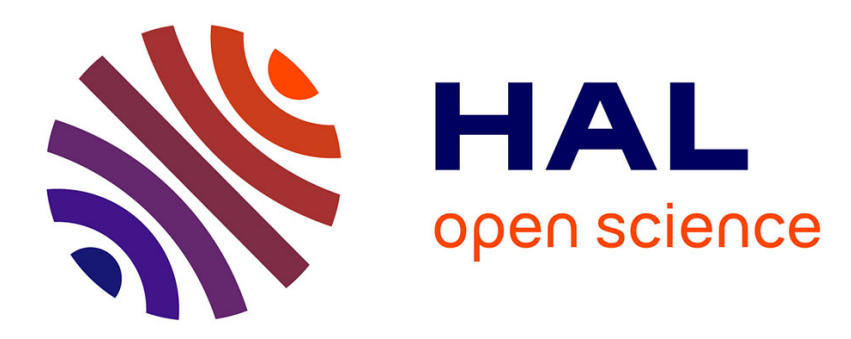

\title{
Utiliser les réseaux virtuels pour renforcer la proximité avec le client
}

Nathalie Audigier

\section{To cite this version:}

Nathalie Audigier. Utiliser les réseaux virtuels pour renforcer la proximité avec le client. Gestion HEC Montréal, 2014. hal-01570920

\section{HAL Id: hal-01570920 \\ https://hal.science/hal-01570920}

Submitted on 1 Aug 2017

HAL is a multi-disciplinary open access archive for the deposit and dissemination of scientific research documents, whether they are published or not. The documents may come from teaching and research institutions in France or abroad, or from public or private research centers.
L'archive ouverte pluridisciplinaire HAL, est destinée au dépôt et à la diffusion de documents scientifiques de niveau recherche, publiés ou non, émanant des établissements d'enseignement et de recherche français ou étrangers, des laboratoires publics ou privés. 


\section{$\underline{\text { Utiliser les réseaux virtuels pour renforcer la proximité avec le client }}$}

\section{Nathalie Audigier}

Nathalie Audigier est maître de conférences en gestion à l'Université de Bretagne-Sud, nathalie.audigier@univ-ubs.fr. 


\title{
Résumé
}

Un besoin d'interactivité se manifeste chez les consommateurs, ce qui traduit de fortes attentes en termes de proximité relationnelle, de convivialité et d'identification mutuelle. Les entreprises et les marques veulent devenir auprès des individus de véritables référents à partir de leur propre univers sémantique. Cependant, maîtriser l'interactivité numérique et «accrocher» le consommateur implique des choix dans les options de communication et l'adoption de pratiques de gestion spécifiques pour la gestion de la relation client.

Mots clés : interactivité, consommateur, marque, communication commerciale, communication virtuelle

\begin{abstract}
$\underline{\text { Abstract }}$
A need for interactivity shows itself at the consumer's, translating waits strong in terms of relational closeness, user-friendliness and mutual identification. Companies and marks (brands) want to become with the individuals of real referents from their own semantic universe. However, master the digital interactivity and «hang on» the consumer involve choices in the options of communication and the adoption of specific managerial practices for the customer relationship management.
\end{abstract}

Keys words : interactivity, consumer, brand, business communication, virtual communication 


\section{$\underline{\text { Utiliser les réseaux virtuels pour renforcer la proximité avec le client }}$ \\ Nathalie Audigier}

Nous assistons aujourd'hui à la mise en relation et en réseaux d'outils et de technologies qui, jusqu'alors, se développaient de façon isolée. Sur le plan entrepreneurial, cela implique la définition de nouvelles stratégies d'accroche et de conquête du consommateur à travers notamment une approche novatrice dans les choix du marketing opérationnel.

Pour le consommateur, il s'agit d'une nouvelle façon d'appréhender le monde : la proximité technologique provoque un surinvestissement dans l'immédiat sans prise de recul, sans esprit critique, où, en face de son écran, l'internaute est à la recherche de satisfactions partielles et momentanées ${ }^{1}$.

Sur le plan sociologique, cela implique une mutation des modes d'insertion des individus dans les communautés humaines et la remise en question du concept même de lieu de vie lorsque l'on peut se connecter aux quatre coins du monde, acheter au sein de galeries marchandes virtuelles, et ainsi de suite. Les nouvelles technologies de l'information et de la communication (NTIC) ont généré de multiples options dans le rattachement à un groupe d'appartenance, à travers l'apparition de dizaines de formes et de «lieux de communautés à géométrie variable et à responsabilités limitées ${ }^{2} »$. Nous entendons par «groupe d'appartenance» un ensemble de personnes qui ont des caractéristiques similaires, qui possèdent leurs propres normes et incitent leurs membres à respecter les croyances et les «lois» du groupe. Au sein des groupes d'appartenance, on peut distinguer deux grandes catégories ${ }^{3}:$ les groupes primaires, avec lesquels une personne a des relations directes et fréquentes (famille, amis, camarades de classe, etc.), et les groupes secondaires, avec lesquels les relations sont moins fréquentes et en général indirectes (associations, clubs, parti politique, etc.). Dans cet article, nous examinons, dans un premier temps, les principaux changements qu'entraîne l'usage du numérique dans les relations entre les marques et les 
consommateurs, avant de présenter, dans un second temps, des options de gestion qui peuvent être intégrées dans les stratégies des entreprises.

\section{La numérisation des échanges : les relations entreprises-clients changent de forme et de fond}

$\mathrm{Au}$ niveau international, nous remarquons le développement d'une économie de l'information ou économie de l'immatériel, qui se traduit par une croissance des services en ligne et s'appuie sur une relation interactive avec le consommateur.

\section{L'émergence des leaders d'opinion}

L'économie de l'information engendre de nouveaux modes d'organisation en estompant les frontières entre métiers, secteurs d'activité, en déstabilisant les anciennes hiérarchies et en renforçant les mouvements de concentration, faisant émerger des leaders d'opinion. On constate notamment une inversion du schéma de communication : l'annonceur perd l'initiative de la communication au profit de la cible, soit les clients, lesquels élargissent l'auditoire des messages au sein de forums.

L'observation de certains groupes virtuels montre que des individus interviennent plus fréquemment et plus durablement que d'autres, ont des échanges avec un plus grand nombre d'internautes ${ }^{4}$. Notre étude révèle également que l'utilisation de plus en plus importante d'Internet et des réseaux virtuels favorise l'apparition d'un porte-voix, qui va «personnifier» les avis, comme si l'immatérialité des échanges trouvait dans l'apparition d'un leader un palliatif pour humaniser les prescriptions.

L'identification des leaders d'opinion est un processus complexe qui trouve son origine dans un ouvrage publié en 1955 par Katz et Lazarsfeld. Ceux-ci identifient le leader d'opinion selon trois dimensions : «qui suis-je?» (traits de personnalité), «qu'est-ce que je connais?»(domaine d'expertise) et «quelles personnes je connais?» (position et visibilité sociale). Les recherches axées sur l'identification des leaders d'opinion sont issues essentiellement des sciences politiques et du marketing. Ce processus d'identification est 
considéré comme long et fastidieux, mais ô combien utile dans les différents champs disciplinaires. Par ailleurs, les techniques d'identification peuvent varier selon le contexte. En effet, qu'on soit en face d'un produit technique, d'un produit alimentaire ou encore d'un service, les caractéristiques propres aux leaders seront différentes. L'expertise qui devrait figurer parmi les caractéristiques du leader n'est pas toujours sa force, et certains internautes s'imposent dans ce statut, grâce à leurs qualités de communicants (savoir gérer leur image et leur discours), à leur capital image (personnage people).

Une autre dimension à considérer concernant les leaders d'opinion, c'est le fait qu'ils sont sollicités davantage par leur entourage. Selon une étude parue dans la revue eMarketer, $76 \%$ des consommateurs sollicitent l'avis d'un ami avant d'acheter un nouveau produit, $68 \%$ prennent en compte une précédente expérience vécue avec l'entreprise, $22 \%$ lisent des recommandations publiées dans les journaux ou dans les magazines, $15 \%$ font confiance à la publicité et $8 \%$ consultent les informations disponibles sur le site Web de l'entreprise. Les entretiens menés auprès des internautes (voir l'encadré 1) permettent de mesurer l'importance de la communication virtuelle.

\section{Encadré 1 - À propos de l'étude}

Nous avons mené, en France, de septembre 2012 à mars 2013, une trentaine d'entretiens semi-directifs par téléphone : 18 internautes appartenant à divers réseaux sociaux et 12 responsables du marketing ou de la communication au sein d'entreprises, représentant différents secteurs d'activité. À partir d'un guide d'entretien, nous avons tenté de comprendre les liens recherchés par les uns et par les autres à travers l'utilisation des médias en ligne et la manière dont se construisait la communication virtuelle, tant pour l'annonceur (l'entreprise) que pour la cible (le client).

Cet aspect de la sollicitation est à mettre en relation avec les notions de crédibilité et d'impartialité. Plus la crédibilité est grande, plus l'influence est forte. Autrement dit, les leaders d'opinion sont sollicités par les communautés virtuelles parce qu'ils apparaissent comme crédibles et impartiaux : leurs conseils sont 
désintéressés, contrairement à la publicité. Dans cette logique, pour que le leader d'opinion soit opérationnel, il doit être déconnecté le plus possible de l'entreprise, qui doit donc accepter d'abandonner à sa cible une partie de la maîtrise de sa communication.

\section{Les clients à la fois cibles et responsables de la communication}

Dans cette nouvelle logique économique, nous relevons différents facteurs rapprochant l'internaute de l'univers des entreprises. Avec la création de communautés marchandes et non marchandes, l'internaute est présent au sein même des entreprises grâce aux caractéristiques propres d'Internet (connectivité, ubiquité, fluidité, rapidité et universalité). Par ailleurs, la relative transparence de ce «cybermonde» est renforcée par l'accès à une information plus large et plus disponible pour l'usager-consommateur; il faut toutefois nuancer cet aspect lorsque l'utilisateur est peu expérimenté et se trouve perdu dans les méandres du Web.

L'individu peut et veut devenir un véritable «consommacteur» en s'intégrant toujours davantage dans la chaîne de valeur de l'organisation. L'utilisateur final de l'information cherche de plus en plus à être actif et non plus seulement réactif - dans la création de cette information ${ }^{5}$. Les réponses obtenues dans le cadre de nos entretiens avec les internautes montrent qu'ils ne se considèrent pas comme des «cibles» des messages, mais comme des participants plus ou moins actifs à la construction des messages.

Le Web 2.0, en tant qu'espace public mondial de création et d'innovation sur Internet, illustre bien l'intervention croissante des individus dans l'innovation technologique qui devient «ascendante» ${ }^{6}$. Cette constatation coïncide avec le fait qu'une nouvelle génération née dans les années 1980 et 1990 a grandi avec le phénomène numérique : elle ne peut concevoir de vivre sans Internet, le téléphone portable ou le MP3. Dans cette économie de l'immatériel, le consommateur ou l'usager est également coproducteur de l'information, puisqu'en l'utilisant il lui donne sa véritable valeur ajoutée. 


\section{$\underline{\text { Implications de gestion pour les entreprises }}$}

L'apparition des leaders d'opinion sur les réseaux et les forums ainsi que la participation plus grande des internautes à la communication des entreprises représentent des risques pour l'annonceur, qui perd en partie la responsabilité et la maîtrise de son message. Les entreprises interrogées se félicitent de l'intérêt que suscite leur présence sur la Toile, mais elles redoutent en même temps de ne plus pouvoir contrôler ce qui est dit les concernant.

À cela il est possible d'ajouter que le développement du numérique crée une interactivité électronique qui modifie le modèle de représentation du monde et pose ainsi le problème du cadre d'interprétation de l'information. En effet, il n'y a plus de sources reconnues et fiables : l'émetteur premier s'efface progressivement pour n'apparaître que comme le relais d'une information sans signataire, ce qui pose le problème de l'authentification de tout ce qui circule sur les réseaux. Comme l'énonçait Cartier en 2004, pour l'individu qui est à la recherche à la fois de connaissances et de participation, le défi n'est plus de diffuser et de fournir des milliards de pages, mais de leur donner un sens.

Dans ce nouveau contexte, nous proposons aux responsables d'entreprise des options de gestion qui leur permettront de garder la maîtrise de leur communication de marque ainsi que des leviers d'actions qui les aideront à renforcer la relation client-marque.

\section{Garder la maîtrise de la communication de marque}

La marque commerciale est devenue l'un des principaux vecteurs de l'image et de la responsabilité sociale de l'entreprise. Elle devient «le signe de l'entreprise qui l'oblige à répondre aux exigences du citoyen autant que du consommateur ${ }^{7} \gg$.

Par l'interface de la marque se crée une relation inédite entre les consommateurs et l'entreprise. En effet, nous assistons à l'évolution d'une logique de marque normative présentant, voire imposant, un univers de 
vie au consommateur vers une logique de marque modelable qui va intégrer et respecter les codes du consommateur afin de mieux le séduire. La marque commerciale, principal actif immatériel de l'entreprise, peut être, de ce fait, plus facilement discutée et critiquée soit directement par le site Internet de l'entreprise ou de la marque, soit indirectement par le truchement des sites ou des blogues de groupes de pression (les associations de consommateurs, les écologistes, les organisations non gouvernementales, etc.).

Notre étude exploratoire indique que les internautes sont adeptes du plaidoyer, à savoir qu'ils prennent volontiers position en faveur d'une marque, d'une idée dont ils se font les avocats ou les ambassadeurs auprès de leur entourage et de leur communauté virtuelle. Toutefois, on note que les médias sociaux ne sont que le miroir d'un bouche-à-oreille qui existait avant, la tonalité des propos restant la même, la vraie différence se situant dans l'espace de diffusion des avis. Les sujets les plus évoqués sur les réseaux sociaux sont ceux qui suscitent le plus d'intérêt et qui agacent le plus : la qualité, le prix des produits et des services, la fiabilité des offres commerciales, l'accueil ménagé aux clients, etc. Les thématiques sociétales sont moins présentes dans les débats. De façon générale, les prises de positions citoyennes mobilisent moins de monde que les bons plans ; les sujets liés à la responsabilité sociale de l'entreprise ou à l'éthique bénéficient d'un retentissement plus limité et globalement plus négatif comparativement aux sujets commerciaux. Par exemple, en France, les internautes seront plus nombreux à critiquer un bon plan proposé par un distributeur comme Leclerc que lorsque l'assureur MAIF fait une communication sur son engagement humanitaire. Pour générer des ambassadeurs sur les réseaux sociaux, il faut un message sincère et crédible qui combine l'intérêt collectif avec le bénéfice individuel (l'axe de communication de La Poste).

Les marques commerciales doivent donc élaborer sur leurs blogues ou leurs forums de discussion un discours transparent, honnête, alliant une proximité relationnelle et une expertise certaine dans leurs secteurs d'activité. L'usage du numérique par le consommateur traduit de fortes attentes en termes de 
proximité relationnelle, de convivialité et d'identification mutuelle. Internet expose les marques au regard de la société, mais il contribue également à une meilleure compréhension de celles-ci. Les marques associées à Internet peuvent fidéliser leurs clients si elles sont capables de renouveler constamment leur offre en cohérence avec le système de valeurs de ces derniers. Comme le déclare Baudoin (2006), les internautes «sont fidèles à leur impatience»; ils peuvent de ce fait se connecter régulièrement à une source si celle-ci est capable d'innover en permanence dans le respect de leurs attentes.

Le fait de maîtriser et de développer l'interactivité de la marque et de ses clients actuels ou potentiels, notamment à l'égard de la communication commerciale, nécessite non seulement des capacités d'investissement sur les plans financier et technologique, mais surtout une aptitude à répondre aux nouvelles attentes socioculturelles des consommateurs (une réactivité par rapport au contenu des échanges sur les forums, des réponses aux questions posées aux services à la clientèle en ligne, etc.).

\section{Renforcer la relation client-marque en trois actions}

L'exploitation des résultats de notre étude qualitative nous permet de distinguer trois voies d'actions principales conditionnant le succès de la relation interactive client-marque (voir le schéma 1).

\section{Schéma 1 - Actions pour favoriser la relation client-marque}

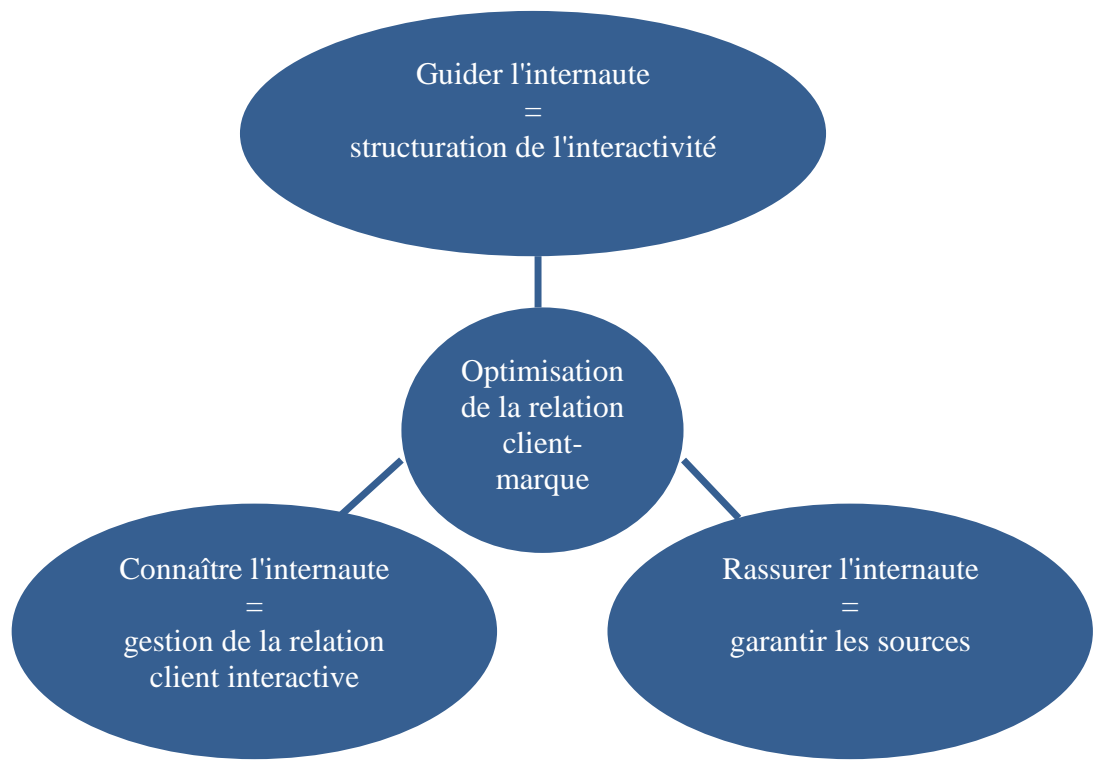


Action 1 : guider l'internaute en structurant l'interactivité commerciale. On peut constater sur Internet un paradoxe apparent entre une liberté d'explorer, de découvrir sans frontières et, en même temps, une forte attente de simplification, d'organisation et de rationalisation du réseau pour les consommateurs. Même si l'utopie du réseau interactif consiste en la libre recherche d'information sur les «richesses de la planète», on observe un besoin croissant d'être guidé au fur et à mesure que les réseaux s'étendent et se complexifient.

L'entreprise doit alors proposer des procédures, des services personnalisés d'orientation, de recherche et de sélection de données utiles au client. De plus, les travaux de Holbrook et Hirschman, en 1982, ont conduit à l'élaboration d'un modèle «expérientiel» qui met l'accent sur l'importance de la dimension affective dans le comportement du consommateur. La consommation représente une expérience pour un individu, et cette expérience peut susciter toute la gamme des réactions affectives ${ }^{8}$. Les individus ont, de ce fait, de nouvelles attentes à l'égard des marques. L'image du statut social, attachée à une marque ou à la seule habitude d'achat, est devenue insuffisante pour garantir la fidélité. Les individus sont devenus des consommateurs zappeurs. Les entreprises et leurs marques qui veulent s'inscrire dans la vie des consommateurs, de leur famille et de leur tribu doivent savoir s'appuyer sur des valeurs susceptibles de créer des émotions afin de construire leur image et leur personnalité et de se différencier des concurrents. La valeur ajoutée «émotionnelle» d'une marque permet d'élaborer un véritable engagement relationnel et éthique entre le consommateur et les organisations.

Les entreprises ont intérêt à mettre au point des solutions de marketing mobile, permettant aux marques d'être connectées directement à leurs consommateurs individuellement, sans contraintes de temps ou d'espace. Le marketing mobile va d'ailleurs de plus en plus loin dans la personnalisation des offres en prenant progressivement en compte la localisation des consommateurs permise par les nouvelles technologies telles que le GPS intégré dans les téléphones intelligents. Internet permet aussi de recueillir de nouvelles données comportementales virtuelles, qui sont les relations et les interactions d'un 
consommateur avec un site Internet. En effet, outre les données qu'un site peut amasser au moment d'une inscription, les nouveaux moyens d'analyse sur Internet permettent, à partir de chemins de navigation, d'établir des profils de consommateurs, ce qui donne aux responsables du marketing en ligne la possibilité d'adapter automatiquement les offres en fonction des préférences et des comportements des consommateurs et de garder ainsi le contrôle de la relation commerciale.

Action 2 : rassurer l'internaute en garantissant la source des informations. Les réseaux de communication numérique concurrencent les médias de masse en multipliant les sources de production d'information et en mondialisant les échanges. Chaque consommateur peut devenir «proactif», c'est-à-dire concepteur, catalyseur, émetteur et récepteur d'un message. Cette situation où chaque individu est susceptible de devenir un média peut générer un problème d'évaluation plus difficile de la crédibilité des informations et des messages. De ce fait, l'entreprise doit être capable de garantir les informations qu'elle transmet au même titre que la sécurité des paiements. La crédibilité que l'on prête à une certification, à un label socioprofessionnel, à un titre ou à une signature manuscrite doit trouver l'équivalent en matière électronique. Dans ce contexte, les marques ayant une forte image peuvent disposer d'un avantage concurrentiel réel en matière de garantie et de crédibilité des messages émis à leurs clients actuels ou potentiels.

Cova (1996) va plus loin en établissant que le consommateur «souhaite aujourd'hui un marketing, fondé sur l'expérience, qui favorise les relations interpersonnelles et la créativité». Kacen (2000) et Dawes et Brown (2000) montrent également que dans ce contexte postmoderne, les marchés sont de plus en plus fragmentés et nécessitent une adaptation des outils de marketing. En effet, les consommateurs, dans leurs recherches identitaires, tentent d'adapter et de construire de nouveaux styles, de nouvelles formes à partir des produits et des marques, devenant ainsi autant créateurs que consommateurs. Les responsables du marketing doivent donc aujourd'hui avoir en tête cette nouvelle donne : la production est indissociable de la consommation. Internet et les outils de personnalisation, de partage et de création en ligne permettent 
cette coproduction des consommateurs avec les marques, donnant toute liberté aux consommateurs postmodernes d'exercer leur pouvoir, d'imposer leurs choix et leurs désirs face aux marques.

Action 3 : améliorer la connaissance du client par une gestion de la relation client interactive. La collecte des informations et la gestion des mégabases de données permettront de répondre aux aspirations individualisées du consommateur, de mettre en œuvre une production «sur mesure», d'assurer la distribution et la vente directe des produits et des services ainsi que de créer une véritable relation de communication personnalisée avec un consommateur qui peut apparaître insaisissable. Le tableau 1 présente les modalités opérationnelles pour construire la relation marque-client. Traditionnellement, trois «V» définissent les bases de données : le «volume» (la masse de données), la «variété» (des données de natures diverses : vidéos, photos, textes, etc.) et la «vélocité» (les données à gérer en temps réel), auxquels il faut ajouter un quatrième «V» pour «valeur», car les données de masse représentent un levier d'affaires puissant à condition qu'il soit bien maîtrisé : cela renvoie à la capacité de l'entreprise de stocker des données, de les trier, de les analyser et de les intégrer dans son modèle d'affaires (voir le tableau 1). Il s'agit d'un défi complexe pour les décideurs, puisque, selon les responsables interrogés, seulement $20 \%$ à $30 \%$ de ces données sont utilisées. Plusieurs raisons sont à l'origine de cette déperdition. Tout d'abord, si près de 7 décideurs sur 10 concernés par les bases de données indiquent qu'ils en ont une bonne connaissance, les faits montrent que leur niveau de compréhension des technologies associées reste plutôt faible. 
Tableau 1 - Principes d'une relation client-marque interactive

\begin{tabular}{|l|l|}
\hline \multicolumn{1}{|c|}{ Principes } & \multicolumn{1}{c|}{ Modalités } \\
\hline Attraction & $\begin{array}{l}\text { Établir avec les clients des liens interactifs avec la } \\
\text { marque, permettant de mieux connaître et satisfaire leurs } \\
\text { besoins et désirs individuels }\end{array}$ \\
\hline Différenciation & $\begin{array}{l}\text { Distinguer ses clients à partir de deux critères essentiels - } \\
\text { les besoins et désirs qu'ils expriment par rapport à la } \\
\text { marque ainsi que la valeur économique qu'ils } \\
\text { représentent }- \text { pour découvrir leurs motivations d'achat et } \\
\text { le profit potentiel de chaque client }\end{array}$ \\
\hline Relation d'apprentissage & $\begin{array}{l}\text { Tisser une relation privilégiée entre la marque et le client } \\
\text { pour inciter ce dernier à donner des informations sur son } \\
\text { mode de vie, ses attentes et ses habitudes de } \\
\text { consommation }\end{array}$ \\
\hline Valeur stratégique du client & $\begin{array}{l}\text { Estimer le chiffre d'affaires potentiel qu'un client peut } \\
\text { apporter à une entreprise ou à une marque et déployer des } \\
\text { efforts différenciés en fonction du potentiel que } \\
\text { représente chaque client }\end{array}$ \\
\hline
\end{tabular}

Nos résultats rejoignent en ce sens une étude de Markess International menée en 2012 qui indique que si

$31 \%$ des décideurs engagés dans cette problématique ont poursuivi une réflexion sur le sujet dans leurs organisations, seuls $6 \%$ ont concrètement lancé des projets liés aux bases de données et moins de $5 \%$ des entreprises utilisent des technologies Hadoop (un modèle de programmation code source libre de référence conçu pour traiter de très grands volumes de données, comme Apache Hive, Hadoop File System ou NoSQL). Par ailleurs, les entreprises restent peu nombreuses à utiliser des bases de données en mémoire, qui servent notamment à accélérer les temps de traitement des informations. L'importance d'une connaissance des comportements du consommateur en temps réel doit inciter les entreprises à investir dans des solutions de gestion de la relation client interactives (des solutions SAS, par exemple) pour optimiser leurs chances de satisfaire et de fidéliser leurs clients. 


\section{$\underline{\text { Conclusion }}$}

La rapidité avec laquelle se développent les NTIC nous donne à penser que la veille et l'adaptabilité sont indispensables non seulement du côté des technologies, mais aussi du côté des usages, des pratiques et des compétences que met en œuvre l'entreprise.

La communication avec les clients-acteurs doit être basée sur l'écoute et la confiance, ce qui permet de prévenir plutôt que de guérir. Mais si un incident se produit, il est nécessaire d'être présent, d'avoir un lieu technique qui permette au client mécontent de pouvoir immédiatement s'exprimer et obtenir un traitement adapté de sa réclamation. D'autre part, par leur présence sur Internet, les marques cherchent à établir la relation la plus individualisée possible avec leurs consommateurs. En référence aux travaux de Cova (1997), nous préconisons de développer la notion de «valeur lien» (linking value), notamment pour les marques ayant particulièrement vocation à agir à travers des communautés et à s'inscrire comme des supports relationnels pour leurs consommateurs. La marque peut aussi prendre en compte, pour établir son discours, non seulement les attentes vis-à-vis des produits, mais aussi les aspirations émotionnelles et culturelles des individus, de manière à créer une véritable relation entre la marque et la personne.

La relation en ligne est un facteur clé de succès de la stratégie de communication des entreprises qui doivent et devront gérer les rapports à la notion d'image, de valeur ajoutée émotionnelle de leurs marques. La stratégie marketing de l'entreprise et la communication commerciale qui en découle doivent intégrer un consommateur, qui, tel Janus, est à la fois cible et pilote. 


\section{$\underline{\text { Références }}$}

Baudoin, J.-P. (2006), «Marques \& TIC, quelle vitalité!», La Revue des Marques, n 54, avril, p. 6-8.

Brée, J. (2004), Le comportement du consommateur, Dunod.

Cartier, M. (2004), Un procédurier pour le nouveau Web, [En ligne], www.michelcartier.com (Page consultée le 28 septembre 2004).

Cathelat, B. (1998), De l'Homo Sapiens à l'homme interactif, FORESEEN, Plon.

Cova , B (1996) «Les particules expérientielles de la quête d'authenticité du consommateur », Décisions Marketing, 28, pp.33-42.

Cova B. (1997) « Community and consumption: Towards a definition of the "linking value" of product or services», European Journal of Marketing, Vol. 31 Iss: 3/4, pp.297 - 316

Dawes, J. et Brown, RB. (2000) « Postmodern marketing: research issues for retail financial services», Qualitative Market Research: An International Journal, Vol. 3 Iss: 2, pp.90 - 99

Derbaix, C., Pham, M.T. (1989), «Pour un développement des mesures de l'affectif en marketing synthèse des pré-requis», Recherche et Applications en Marketing, vol. 4, n 4, p. 71-87.

Graillot, L. (1998), «Émotions et comportement du consommateur», Recherche et Applications en Marketing, vol. 13, $\mathrm{n}^{\circ} 1$, p. 5-23.

Holbrook, M.B., Hirschman, E.C. (1982). «The experiental aspects of consumption : Consumer fantaisies, feelings and fun», The Journal of Consumer Research, vol. 9, $\mathrm{n}^{\circ}$ 2, septembre, p. 132-140.

Kacen, JJ. (2000) «Girrl power and boyyy nature: the past,present and paradisal future of consumer gender identity» Marketing Intelligence and Planning, 18(6/7), p 345-355

Katz, E., Lazarsfeld, P. (1955), Personal Influence, The Free Press.

Lupiéri, S. (2004), «Faut-il encore croire au progrès?», Enjeux. Les Échos, n 200, spécial anniversaire, mars.

Michon, C. (2006), Le marketeur ,Editions Pearsons

Musso, P., Ponthou, L., Seulliet, E. (2007), Fabriquer le futur 2, Le Village Mondial, Éditions Pearsons Éducation.

Tillinac, J. (2006), «Le Web 2.0 ou l'avènement du client ouvrier», Revue Quaderni, ${ }^{\circ}$ 60, printemps, p. $19-24$.

Vernette, E. (2006), «Une nouvelle vision du leader d'opinion en marketing : une approche phénoménologique», 6 e Congrès international sur les tendances en marketing, Venise, EAP/ESCP (actes sur CD-ROM). 


\section{Notes}

${ }^{1}$ Lupiéri (2004).

${ }^{2}$ Cathelat (1998).

${ }^{3}$ Michon (2006), Brée (2004).

${ }^{4}$ Vernette (2006).

${ }^{5}$ Musso et al. (2007).

${ }^{6}$ Tillinac (2006).

${ }^{7}$ Baudoin (2006).

${ }^{8}$ Derbaix et Pham (1989), Graillot (1998). 